\title{
A PERFORMANCE COMPARISON OF FULLBAND AND DIFFERENT SUBBAND ADAPTIVE EQUALISERS
}

\author{
Hafizal Mohamad ${ }^{1}$, Stephan Weiss ${ }^{1}$, Markus Rupp ${ }^{2}$, and Lajos Hanzo ${ }^{1}$ \\ ${ }^{1}$ Dept. Electronics \& Computer Science, University of Southampton, UK \\ ${ }^{2}$ Wireless Research Lab / Bell-Labs, Lucent Technologies, Holmdel, NJ, USA \\ $\{$ hm99r,sw1,1h\}@ecs.soton.ac.uk, rupp@lucent.com
}

\begin{abstract}
We present two different fractionally spaced (FS) equalisers based on subband methods, with the aim of reducing the computational complexity and increasing the convergence rate of a standard fullband FS equaliser. This is achieved by operating in decimated subbands at a considerably lower update rate and by exploiting the prewhitening effect that a filter bank has on the considerable spectral dynamics of a signal received through a severely distorting channel. The two presented subband structures differ in their level of realising the feedforward and feedback part of the equaliser in the subband domain, with distinct impacts on the updating. Simulation results pinpoint the faster convergence at lower cost for the proposed subband equalisers.
\end{abstract}

\section{INTRODUCTION}

Linear channel distortions caused by multipath propagation and limited bandwidth lead to inter-symbol interference (ISI) at the receiver, which in many cases results in a high bit error rate in the detection. Therefore, many different adaptive equalisation structures have been proposed in the past in order to compensate for these channel distortions in the receiver. Most popular amongst the subset of linear or minimum-mean-square error (MMSE) equalisers are currently fractionally spaced (FS) architectures [1], whereby the equalisation filter operates at a rate higher than the symbol rate.

A standard fractionally spaced equaliser is shown in Fig. 1. The structure operates the feedforward (FF) part of the equaliser at an oversampled rate, here twice the symbol rate. In the flow graph in Fig. 1, the FF part is implemented as a polyphase structure [2] the two polyphase components running $a_{0}[n]$ and $a_{1}[n]$ of the adaptive FF filter at the lower symbol rate. The two filters $a_{0}[n]$ and $a_{1}[n]$ are excited by the two polyphase components of the oversampled channel output $x[m]$. The feedback (FB) part of the equaliser is symbol spaced. This is due to the equation error formulation or the decision feedback mode of the equaliser. In the FB part, the adaptive filter $b[n]$ can be excited by either a training signal (switch position 1) - a copy of the transmitted symbol sequence $u[n]$ delayed by $\Delta$ periods - or in decision feedback mode (switch position 2). All FF and FB parts $a_{0}[n], a_{1}[n]$ and $b[n]$ are adaptive and updated by a suitable algorithm at the symbol rate based on an appropriate criterion of the equalisation error $e[n]$.

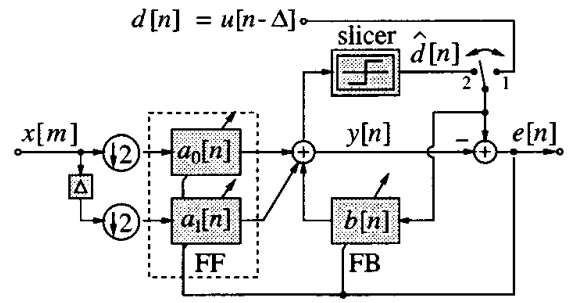

Figure 1: Fractionally spaced equaliser with a polyphase representation of the FF part.

A fractionally spaced equaliser may suffer from considerable computational complexity due to the requirement for long filters if the channel exhibits severe distortions [3], and from slow convergence due to strong spectral dynamics at the input to the equaliser [4]. These characteristics have previously triggered the application of subband techniques to FS equalisers [5], based on the computational reduction, prewhitening, and parallelisation properties of the subband approach $[6,7,8]$. In this contribution, we evaluate two different subband architectures for FS equalisers. This includes a novel scheme for including the equaliser's feedback section into the subband domain, and the incorporation of decision directed subband equaliser structures to track channel alterations after initial equaliser training.

This paper is organised as follows. In Sec. 2, we briefly describe the channel characteristics and motivate subband decompositions. Then, we introduce the proposed subband adaptive equaliser structures and discuss the complexity issue in Sec. 3. In Sec. 4, we present some simulation results to demonstrate the performance of the subband approach.

\section{CHANNEL CHARACTERISTICS AND SUBBAND DECOMPOSITIONS}

For the popularly applied least mean square (LMS) type algorithm in equalisation, the convergence speed is inversely proportional to the eigenvalue spread of its input signal [9]. In turn the eigenvalue spread of a signal can be approximated by the ratio between the maximum and minimum value of its power spectral density (PSD). As an example for the spectral dynamics that can be encountered, we consider a severely dispersive channel given in Fig. 2 . The 
selected channel with a delay spread of approximately 100 symbol periods exhibits additional spectral zeros that reduce the equaliser convergence performance, and also encompasses the transmit and receive filters, that impose a low-pass characteristic on the PSD.

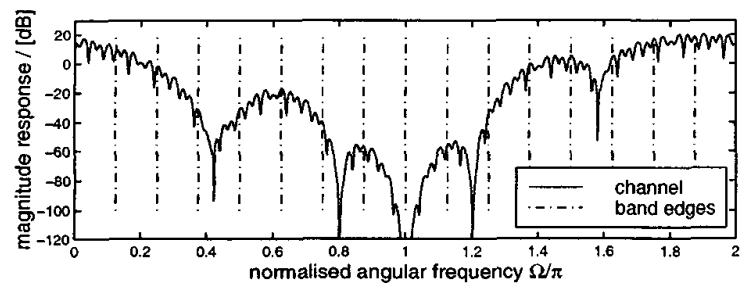

Figure 2: Channel spectral dynamics characteristic with transmit- and receive filter.

A general decomposition into $K$ frequency bands decimated by $N$ (so-called "subbands") is shown in Fig. 3 . The filters in both analysis and synthesis bank are bandpass filters, which, together with the decimation process yield a prewhitening of the subband signals compared to the input. Further, computational savings arise due to an $N$ times lower update rate and lower filter orders compared to fullband implementations. For adaptive filtering applications, adaptive filterings can be operated in each band independently, which lends itself to a parallel implementation. As a drawback, subband structures however introduce aliasing that limits the algorithm performance. Therefore, oversampled filter banks (OSFB) with and oversampling ratio $K / N>1$ are preferred here $[5,6]$. An example of $K=16$ subband channel is indicated by the band edges in Fig. 2, where the eigenvalue spread within each band is reduced. Therefore, the faster convergence of the algorithm is expected with subband decompositions.

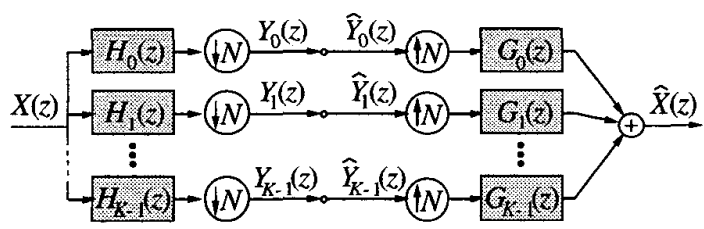

analysis filter bank

synthesis filter bank

Figure 3: $K$-channel filter bank decimated by $N$ with analysis filters $H_{k}(z)$ and synthesis filters $G_{k}(z)$.

An additional benefit of the subband implementation is that an impulse response in the decimated domain can be modelled with less coefficients than required in the fullband case due to the increased sampling period, achieving similar modelling capabilities. In general, this decreases the necessary filter length by a factor of $N$, whereby a moderate overhead of prototype filter coefficients has to be taken into account as in the subband domain potentially fractional delays have to be modelled [7]. The length of subband filter coefficients is given by

$$
L_{\text {Subband }}=\frac{L_{\text {Fullband }}+L_{p}}{N}
$$

where $L_{p}$ denotes for the length of the prototype filter.

\section{SUBBAND ADAPTIVE EQUALISER STRUCTURES}

In this section, we introduce two different subband adaptive equaliser structures and discuss the complexity issues of the equalisers. For the subband implementation, we utilise OSFBs as described in reference [10].

\subsection{Structure I}

For subband equaliser structure I, the FF part of the fullband equaliser in Fig. 1, is projected into subbands. The resulting architecture is shown in Fig. 4, whereby $\mathbf{H}$ and $\mathbf{G}$ denote analysis and synthesis filter bank blocks including decimation and expansion as given in Fig. 3. The system blocks $\mathbf{A}_{0}$ and $\mathbf{A}_{1}$ are diagonal polynomial matrices representing independent filters within each of the $K$ subbands. As the FB part has to be performed at symbol rate, the error is evaluated based on the FF outputs reconstructed by $\mathbf{G}$, and is projected back into the subband domain to update the filters in $\mathbf{A}_{0}$ and $\mathbf{A}_{1}$.

A drawback of the update procedure for the FF part is, that the error signal contains a transfer path. This transfer path can be approximated by a delay identical to $L_{p} / N$. This delay has been reported to result in degraded convergence speed [11]. To overcome this problem, a modification of the structure I architecture will be introduced by integrating the FB part into subbands.

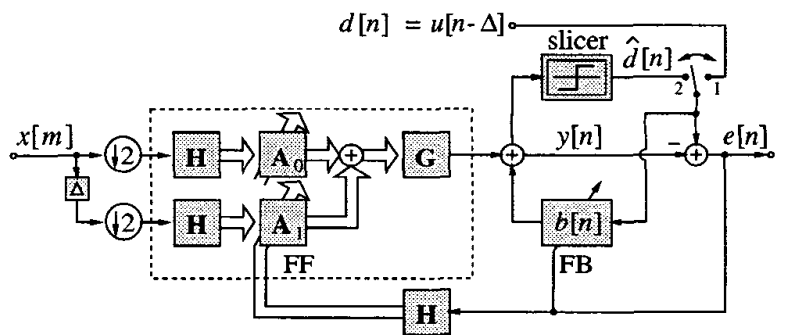

Figure 4: Adaptive equaliser structure I with the FF part in subband.

\subsection{Structure II}

A subband equaliser structure II is shown in Fig. 5, which has the aim to overcome slow convergence due to the error transfer path in structure I. The error signal is now formed in the subband domain and can be used to delaylessly update both the FF and FB parts. Similarly to structure I, $B$ is of diagonal polynomial form holding the adaptive FB filters running independently within each subband.

In structure II architecture, all adaptive filters are updated by the immediately formed subband errors at the 


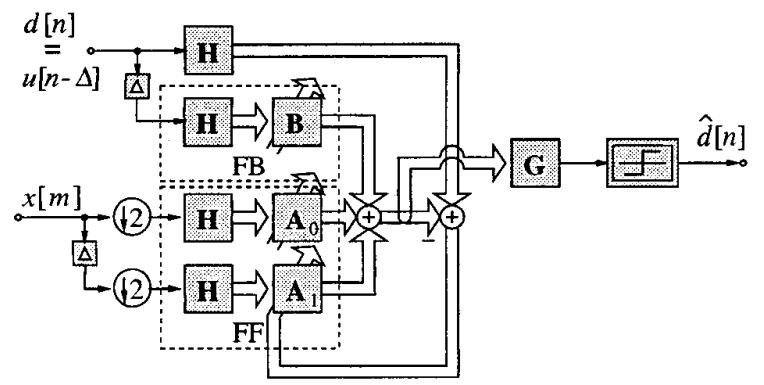

Figure 5: Adaptive equaliser structure II with both the FF and FB parts in subbands.

same time. This is expected to provide improved convergence characteristics over structure I. However, as the error is calculated in the subband domain, this structure can only be used in training mode. The decision directed learning mode - switch position 2 in the fullband structure in Fig. 1 and the subband structure $I$ in Fig. 4 - requires a non-linearity that cannot be transferred into the subband domain. Therefore, if decision directed mode was to be performed, structure I would have to be selected. By appropriate subband projections, the FB filter $b[n]$ in Fig. 4 can be reconstructed from $\mathbf{B}$ in Fig. 5 .

\subsection{Computational Complexity}

The complexity of a fullband equaliser implementation in terms of multiply-accumulates (MACs) when using an NLMS algorithm for updating is approximately given by

$$
C_{\text {fullband }}=4 \cdot 2\left(L_{\mathrm{FF}}+L_{\mathrm{FB}}\right)=8\left(L_{\mathrm{FF}}+L_{\mathrm{FB}}\right)
$$

where the factor of 4 accounts for the required complex valued arithmetic. The feedforward and feedback filter lengths are represented by $L_{\mathrm{FF}}$ and $L_{\mathrm{FB}}$, respectively.

For our subband equaliser implementations, the complexity of the filter banks has to be considered. In a fast implementation, one analysis or synthesis filter bank operation cost

$$
C_{\text {filterbank }}=\frac{1}{N} \cdot\left(2 L_{p}+4 K \log _{2} K\right)
$$

MACs per fullband sampling period [10].

Thus the complexity of subband structure I with the FF part in subband and 4 filter bank operations is

$$
C_{\text {subband }, \mathrm{I}}=\frac{K}{N} 4 \cdot 2\left(L_{\mathrm{FF}}\right)+4 \cdot 2\left(L_{\mathrm{FB}}\right)+4 C_{\text {filterbank }} .
$$

For subband structure II, we require

$$
C_{\text {subband,II }}=\frac{K}{N} 4 \cdot 2\left(L_{\mathrm{FF}}+L_{\mathrm{FB}}\right)+5 C_{\text {filterbank }}
$$

due to operating both FF and FB parts in subbands in the structure and executing 5 filter banks.

\section{SIMULATIONS AND RESULTS}

The channel characteristic in Fig. 2 has been used to test the fullband and subband equalisers introduced in Sec. 3. Quadrature amplitude modulation (QAM) signals are used in our simulation. A normalised least mean square (NLMS) algorithm is employed for adaptation of the fullband and subband structure II adaptive filters, while a delay-NLMS is used in subband structure I. The normalised step size of $\tilde{\mu}=0.4$ is set for all equaliser structures. The delay $\Delta$ for the different systems is set such that the FF part targets almost only the pre-cursor, while the FB part of the equaliser eliminates the post-cursor. For the subband structures, the OSFBs split the fullband signal into $K=16$ channels decimated by $N=14$, with $L_{p}=448$.

The filter length of the subband equalisers is selected according on (1). The number of coefficients of the different structures $-L_{\mathrm{FF}}$ refers to the filter in the FF part, and $L_{\mathrm{FB}}$ to the FB part of the equaliser - is listed in Tab. 1.

\begin{tabular}{|l||c|c|}
\hline Equaliser structure & $L_{\mathrm{FF}}$ & $L_{\mathrm{FB}}$ \\
\hline \hline Fullband & 500 & 100 \\
\hline Structure I & 70 & 100 \\
\hline Structure II & 70 & 40 \\
\hline
\end{tabular}

Table 1: Number of coefficients in the FF and FB parts of the different simulated equaliser structures.

The performance of the three - fullband, and subband structure I and II - equaliser systems is assessed in terms of achieved mean squared error (MSE) and bit error rate (BER), whereby both the learning characteristic as well as the steady state are of interest.

\subsection{Convergence Behaviour}

The MSE learning characteristic of the three systems is presented in Fig. 6. The curves are averaged over an ensemble of 25 runs with a random 64-QAM input signal $u[n]$ in the absence of channel noise. In terms of convergence rate, the subband structures exhibit a convergence speed that is approximately twice as fast as the fullband equaliser. Whereby subband structure II attains a faster initial MSE convergence performance over structure I. It is indicative that both subband structure I and II attain a considerably better steady-state error performance than the fullband system.

\subsection{Bit Error Rates}

We further examine the performance of the fullband equaliser and subband structure II in terms of BER for various levels of QAM over the previous channel, which now is disturbed by noise at variable SNR. The noise is independent of the transmitted signal. An additive white Gaussian noise is coloured by the receive filter. The BER performance results for 4-, 16-, 64-, and 256-QAM over variable SNR are shown in Fig. 7. The displayed BER values are taken for the steady-state case after adapting the equalisers for $5 \cdot 10^{5}$ symbol periods. In general, the fullband equaliser is superior particularly for lower modulation levels at low SNR. 


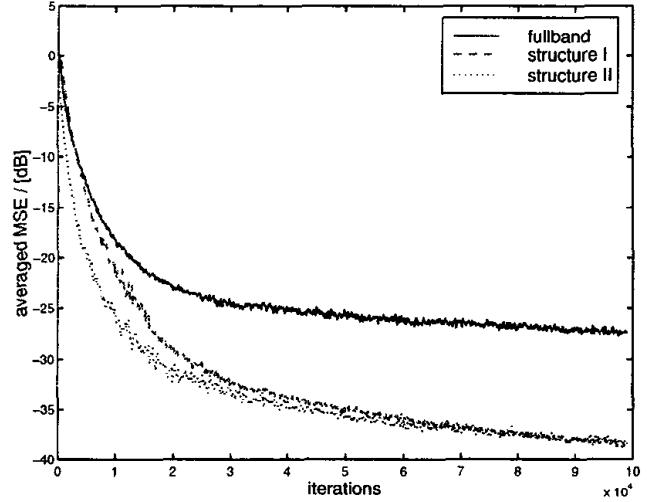

Figure 6: MSE performance for fullband and subband (structure I and II) equalisers for a noise free channel.

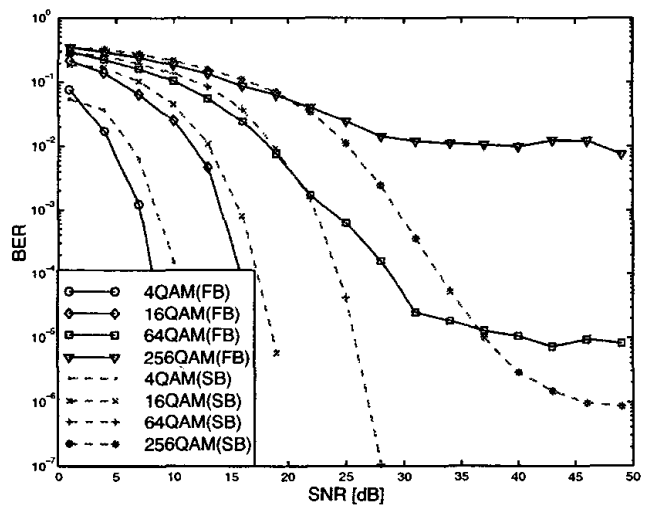

Figure 7: BER performance of fullband (FB) and subband (SB) structure II over variable channel SNR for various modulation levels.

A clear advantage for the steady-state performance of the subband structure can be noted for higher QAM levels (64QAM and 256-QAM) at higher SNR above $25 \mathrm{~dB}$.

\subsection{Computational Cost Comparison}

The filter lengths of the proposed subband structures - selected according to (1) - are given in Tab. 1. These filter lengths have been set to achieve similar modelling capabilities of the fullband and different subband structures. The computational complexity of the equaliser structures - calculated according to (2), (4), and (5) - are displayed in the second column of Tab. 2. The third column in Tab. 2 represents the computational cost comparison for the subband equalisers implementations compared to the fullband realisation. Subband structure I and II only require $39 \%$ and $29 \%$, respectively, of the fullband equaliser's computational complexity.

\begin{tabular}{|l||c|c|}
\hline Equaliser structure & MACs & \% of Fullband \\
\hline \hline Fullband & 4800 & $100 \%$ \\
\hline Structure I & 1882 & $39 \%$ \\
\hline Structure II & 1416 & $29 \%$ \\
\hline
\end{tabular}

Table 2: Computational cost comparison for different equaliser structures.

\section{CONCLUSIONS}

This paper has introduced structures for subband adaptive equalisation and presented some simulation results. An important indication from these results is that for severely distorting channels subband equalisers can attain a faster convergence rate and better steady-state error than their fullband counterpart with a gain in BER for high SNR when operating in higher level QAM modes. The subband equalisers were implemented at a reduced computational cost compared to the fullband system.

\section{REFERENCES}

[1] J. R. Treichler, I. Fijalkow, and C. R. Johnson, "Fractionally Spaced Equalizers: How Long Should They Really Be?," IEEE SP Mag, 13(3):65-81, May 1996.

[2] P. P. Vaidyanathan, Multirate Systems and Filter Banks, Prentice Hall, 1993.

[3] S. Weiss, S. R. Dooley, R. W. Stewart, and A. K. Nandi, "Adaptive Equalization in Oversampled Subbands," IEE Elec. Let., 34(15):1452-1453, July 1998.

[4] M. Rupp, "On the Learning Behaviour of Decision Feedback Equalizers," in Asilomar Conf. SSC, Monterey, CA, Oct. 1999.

[5] S. Weiss, M. Rupp, and L. Hanzo, "A Fractionally Spaced DFE with Subband Decorrelation," in Asilomar Conf. SSC, Monterey, CA, Nov. 2000.

[6] W. Kellermann, "Analysis and Design of Multirate Systems for Cancellation of Acoustical Echoes," in Proc. ICASSP, 5:2570-2573, New York, 1988.

[7] A. Gilloire and M. Vetterli, "Adaptive Filtering in Subbands with Critical Sampling: Analysis, Experiments and Applications to Acoustic Echo Cancellation," IEEE Trans SP, 40(8):1862-1875, Aug. 1992.

[8] J. J. Shynk, "Frequency-Domain and Multirate Adaptive Filtering," IEEE SP Mag., 9(1):14-37, Jan. 1992.

[9] B. Widrow and S. D. Stearns, Adaptive Signal Processing, Prentice Hall, Englewood Cliffs, New York, 1985.

[10] S. Weiss and R. W. Stewart, "Fast Implementation of Oversampled Modulated Filter Banks," IEE Elec. Let., 36(17):1502-1503, Aug. 2000.

[11] M. Rupp and R. Frenzel, "Analysis of LMS and NLMS Algorithms with Delayed Coefficient Update under the Presence of Spherically Invariant Processes," IEEE Trans SP, 42(3):668-672, Mar. 1994. 